\title{
Accuracy and diagnostic performance of doppler echocardiography to estimate mean pulmonary artery pressure in heart failure
}

\author{
Alva Björkman BSc ${ }^{1}$ | Lars H. Lund MD, PhD ${ }^{2} \quad$ Ulrika Ljung Faxén MD, PhD ${ }^{2,3}$ \\ Per Lindquist $\mathrm{PhD}^{4} \quad \mid$ Ashwin Venkateshvaran $\mathrm{PhD}^{2}$ (1)
}

${ }^{1}$ Department of Clinical Physiology,

Karolinska Institutet, Stockholm, Sweden

${ }^{2}$ Department of Medicine, Cardiology Unit, Department of Clinical Physiology, Karolinska Institutet, Stockholm, Sweden

${ }^{3}$ Perioperative Medicine and Intensive Care, Karolinska University Hospital, Stockholm, Sweden

${ }^{4}$ Department of Clinical Physiology, Surgical \& Perioperative sciences, Umeå University, Umeå, Sweden

\section{Correspondence}

Ashwin Venkateshvaran PhD, FASE, FESC, Department of Cardiovascular Research, Karolinska University Hospital, Stockholm 17176, Sweden.

Email: ashwin.venkateshvaran@ki.se

Funding information

Vetenskapsrådet, Grant/Award Numbers: 2013-23897-104604-23, 523-2014-2336

\begin{abstract}
Background: Multiple echocardiographic algorithms have been proposed to estimate mean pulmonary artery pressure $\left(\mathrm{PAP}_{\mathrm{M}}\right)$ and assess pulmonary hypertension ( $\mathrm{PH}$ ) likelihood. We assessed the accuracy of four echocardiographic approaches to estimate $\mathrm{PAP}_{\mathrm{M}}$ in heart failure (HF) patients undergoing near-simultaneous right heart catheterization (RHC), and compared diagnostic performance to identify $\mathrm{PH}$ with recommendation-advised tricuspid regurgitation peak velocity ( $T R V_{\max }$ ).

Methods: We employed four validated echocardiographic algorithms incorporating tricuspid regurgitation peak or mean gradient, pulmonary regurgitation peak gradient, and right ventricular outflow tract acceleration time to estimate $\mathrm{PAP}_{\mathrm{M}}$. Echocardiographic estimates of right atrial pressure were incorporated in all algorithms but one. Association and agreement with invasive $\mathrm{PAP}_{\mathrm{M}}$ were assessed. Diagnostic performance of all algorithms to identify $\mathrm{PH}$ was evaluated and compared with the recommended TRV $\mathrm{max}_{\max }$ cut-off.

Results: In $112 \mathrm{HF}$ patients, all echocardiographic algorithms demonstrated reasonable association $(r=.41-.65 ; p<0.001)$ and good agreement with invasive $\mathrm{PAP}_{\mathrm{M}}$, with relatively lower mean bias and higher precision observed in algorithms that incorporated tricuspid regurgitation peak or mean gradient. All methods demonstrated strong ability to identify PH (AUC $=.70-.80 ; p<0.001$ ) but did not outperform TRV max $_{\text {ax }}$ (AUC $=.84 ; p<0.001$ ). Echocardiographic estimates of right atrial pressure were falsely elevated in $30 \%$ of patients.

Conclusions: Echocardiographic estimates demonstrate reasonable association with invasive $\mathrm{PAP}_{\mathrm{M}}$ and strong ability to identify $\mathrm{PH}$ in HF. However, none of the algorithms outperformed recommendation-advised TRV $\mathrm{Tmax}_{\operatorname{ma}}$. The incremental value of echocardiographic estimates of right atrial pressure may need to be re-evaluated.

KEYWORDS

pulmonary hypertension, right heart catheterization, tricuspid regurgitation peak velocity
\end{abstract}




\section{1 | INTRODUCTION}

Pulmonary hypertension (PH) is common in heart failure (HF) ${ }^{1}$ and is associated with poor prognosis. ${ }^{2}$ Passive downstream elevations in left heart pressures often combined with pulmonary arteriolar remodeling are seen both in HF with preserved (HFpEF) and reduced ejection fraction (HFrEF) and carry therapeutic implications. ${ }^{3} \mathrm{PH}$ is defined as per current recommendations as a mean pulmonary artery pressure $\left(\mathrm{PAP}_{\mathrm{M}}\right) \geq 25 \mathrm{~mm} \mathrm{Hg}$ at rest, measured during right heart catheterization (RHC). ${ }^{4}$ In recent years, expert groups have recommended a revised cut-off as $\mathrm{PAP}_{\mathrm{M}}>20 \mathrm{~mm} \mathrm{Hg}$. ${ }^{5,6}$ Although definite diagnosis necessitates an invasive evaluation of $\mathrm{PAP}_{\mathrm{M}}$, Doppler echocardiography is routinely employed to screen for $\mathrm{PH}$ and evaluate hemodynamic severity during follow-up. Multiple approaches to estimate $\mathrm{PAP}_{\mathrm{M}}$ using echocardiography have been previously proposed. ${ }^{7-13}$ Most algorithms incorporate elements of Doppler analysis obtained from tricuspid regurgitation (TR), 7,10,11,13 pulmonary regurgitation $(\mathrm{PR})^{9}$ or flow across the right ventricular outflow tract (RVOT) ${ }^{8,14}$ into empirical relationships to obtain $\mathrm{PAP}_{M}$. However, the accuracy of these approaches to estimate invasive $\mathrm{PAP}_{\mathrm{M}}$ in the specific setting of HF has not been studied. Further, current ESC recommendations do not advise use of any echocardiographic algorithms to assess PAP $_{M}$ but instead recommend the use of tricuspid regurgitation peak velocity (TRV $\mathrm{Tax}_{\max }$ ) cut-off $>2.8 \mathrm{~m} / \mathrm{sec}$ to assign $\mathrm{PH}$ probability. ${ }^{4}$ Availability of alternative echocardiographic approaches that represent invasive $\mathrm{PAP}_{\mathrm{M}}$ could potentially replace $T R V_{\text {max }}$ during screening, and may even obviate the need for invasive assessment.

With this background, we aimed to study the feasibility and accuracy of four different echocardiographic algorithms to estimate $\mathrm{PAP}_{\mathrm{M}}$ in a retrospective analysis of HF subjects undergoing nearsimultaneous RHC. Further, we wished to compare the diagnostic performance of these algorithms with recommendation-based TRV $V_{\max }$ to identify $\mathrm{PH}$.

\section{2 | METHODS}

\subsection{Study population}

Echocardiographic examinations of consecutive patients with clinically judged HF referred for RHC to the Karolinska University Hospital between 2014 and 2018 were retrospectively reviewed. All subjects were hemodynamically stable during assessment and medical therapy was suitably titrated. Patients in atrial fibrillation or with significant arrhythmias and/or poor echocardiographic image quality precluding accurate measurement were excluded. Thereafter, subjects with isolated pre-capillary alterations on RHC were excluded from the analysis. The study was approved by the local ethics committees (DNR 2008/1695-31) and all patients provided written informed consent.

\section{2 | Echocardiographic evaluation}

All patients underwent comprehensive echocardiography employing a Vivid E9 ultrasound system (GE Ultrasound, Horten, Norway) by a single experienced echocardiographer (AV) in keeping with current recommendations. ${ }^{15} 2 \mathrm{D}$ gray-scale images were acquired at 50-80 frames/sec and Doppler tracings were recorded using a sweep speed of $100 \mathrm{~mm} / \mathrm{sec}$. Three consecutive heart cycles were acquired in sinus rhythm. TR was measured with Continuous wave Doppler, considering the most optimal signal obtained from multiple echocardiographic windows. PR was obtained with Continuous wave Doppler from the parasternal short-axis view at the level of the semi-lunar valves. Right ventricular outflow tract flow was obtained by placing a 5-mm Pulsed Doppler signal in the RVOT just proximal to the pulmonic valve. All images were subsequently exported and analyzed offline (EchoPAC PC, version 11.0.0.0 GE Ultrasound, Waukesha, Wisconsin, USA) by an experienced, credentialed echocardiographer blinded to catheterization data.

A summary of approaches employed to evaluate $\mathrm{PAP}_{\mathrm{M}}$ are illustrated in Figure 1. Broadly, $\mathrm{PAP}_{\mathrm{M}}$ was evaluated using four different algorithms taking into consideration evaluation of $\mathrm{TR}^{7,}{ }^{7,10} \mathrm{PR},{ }^{9}$ and RVOT acceleration time (RVOT ${ }_{A T}$ ). ${ }^{8}$ Applying the approach postulated by Aduen et al., ${ }^{7}$ PAP $_{M}$ was estimated by adding TR mean pressure gradient to recommended estimates of right atrial pressure (RAP) obtained from inferior vena cava (IVC) size and collapsibility. ${ }^{15}$ The second approach by Chemla et al. calculated PAP $_{M}$ from estimated systolic pulmonary artery pressure ( $\mathrm{PAP}_{\mathrm{S}}$ ) obtained by adding the gradient corresponding with peak TR velocity $\left(T R V_{\max }\right)$ to IVC-estimated RAP using the relationship $\mathrm{PAP}_{\mathrm{M}}=.61 \times \mathrm{PAP}_{\mathrm{S}}+2 \mathrm{~mm} \mathrm{Hg} \cdot{ }^{10}$ In the third approach (Abbas and colleagues), $\mathrm{PAP}_{\mathrm{M}}$ was estimated by adding gradients obtained from peak PR velocity to corresponding IVC-estimated RAP. ${ }^{9}$ Finally, in the fourth approach proposed by Dabestani et al., RVOT $_{A T}$ was defined during systole as time in milliseconds from beginning of flow to peak velocity. $\mathrm{PAP}_{M}$ was then calculated as $\mathrm{PAP}_{\mathrm{M}}=90$ - $\left(.62 \times\right.$ RVOT $\left._{\text {AT }}\right)$ when AT < $120 \mathrm{msec}$ and 79 - $\left(.45 \times\right.$ RVOT $\left._{A T}\right)$ when AT $\geq 120$ msec. $^{8}$

\section{3 | Invasive evaluation}

Echocardiographic examinations were followed by RHC within a 1hour period. Pharmacological status was unaltered between echocardiography and catheterization. RHC was performed by experienced operators blinded to echocardiography examinations using a 6F Swan Ganz catheter employing jugular or femoral vein access. After suitable calibration with the zero-level set at the mid-thoracic line, pressure measurements were taken from the right atrium (RA), right ventricle (RV), and pulmonary artery (PA) during end-expiration. Five to 10 cardiac cycles were acquired and all pressure tracings were stored and analyzed offline using a standard hemodynamic software package (WITT Series III, Witt Biomedical Corp., Melbourne, FL, USA). 


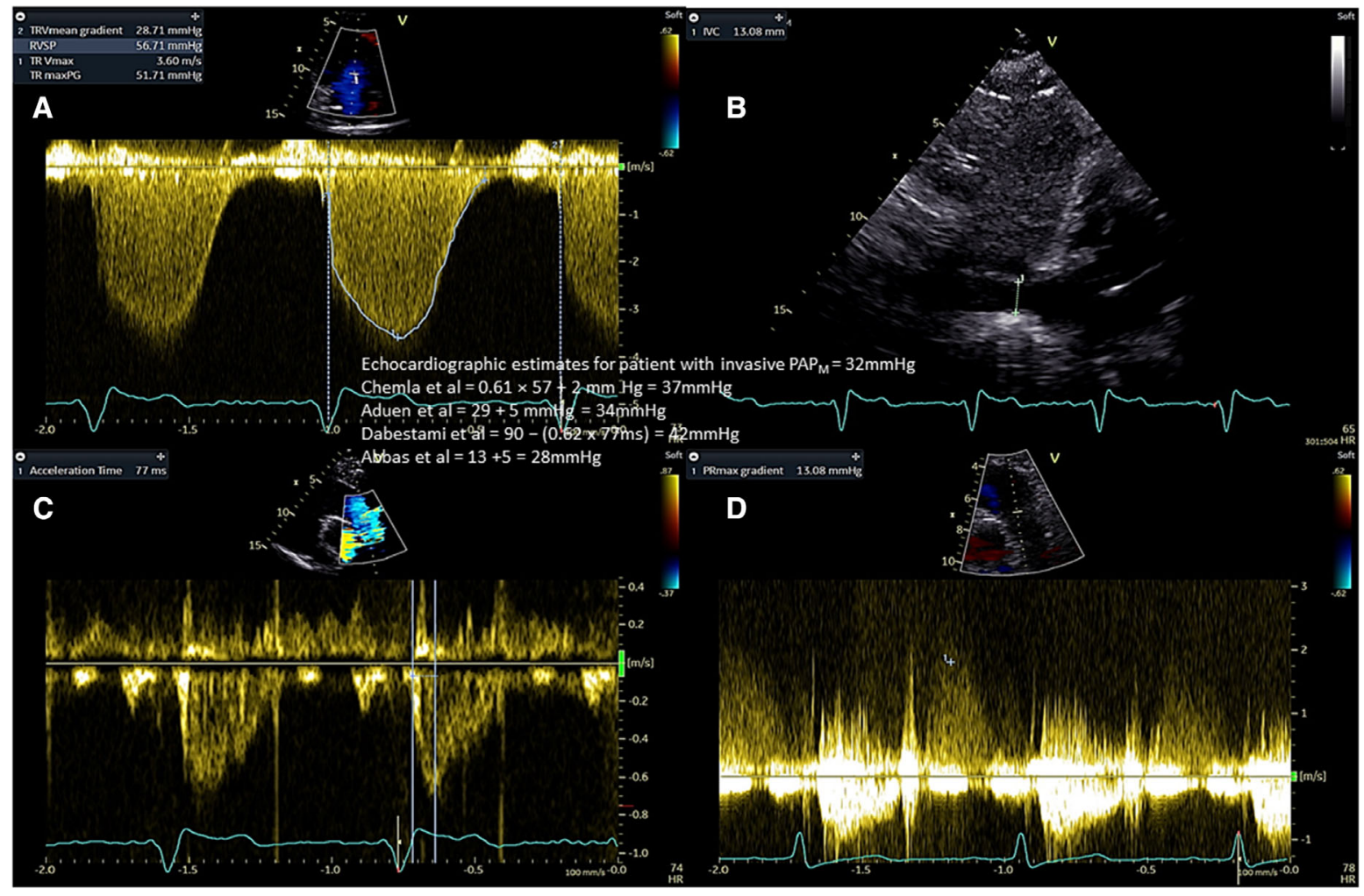

FIGURE 1 Illustration of echocardiographic estimates in a patient with invasive PAPM $=32 \mathrm{~mm} \mathrm{Hg}$. (A) Tricuspid regurgitation CW Doppler

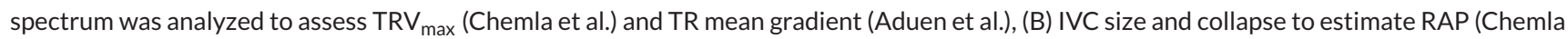
et al., Aduen et al., Abbas et al.), (C) RVOT acceleration time assessed by PW Doppler (Dabestami et al.), (D) PR peak gradient assessed by CW Doppler (Abbas et al.). Resultant echocardiographic estimates for each algorithm are provided

\subsection{Statistical analysis}

Normality was tested using the Shapiro-Wilk test and visually reaffirmed using QQ plots. Continuous variables were expressed as mean \pm SD for parametric variables or median (inter-quartile range) for nonparametric variables and categorical variables were expressed as numbers and percentage. Correlations between Doppler PAP $_{M}$ approaches and corresponding invasive measurements were performed using the Pearson's 2-tailed test (correlation between two continuous variables). Accuracy was defined as the difference of the mean bias and precision as the spread of data points between echocardiographic and invasive measurements on Bland-Altman analysis. Receiver operating characteristics (ROC) curve was employed to illustrate diagnostic potential of both TRV $V_{\text {max }}$ and echocardiographic algorithms. Sensitivity, specificity, negative predictive value (NPV) and positive predictive value (PPV) were measured. IBM SPSS statistics version 23.0 was employed for analysis.

\section{3 | RESULTS}

\section{1 | Study population}

Of 212 enrolled in the study, 45 patients with atrial fibrillation and 46 with pacemaker therapy were first excluded. Nine patients were subse- quently excluded after $\mathrm{RHC}$ revealed isolated pre-capillary alterations. Ultimately, 112 patients ( $60 \pm 16$ years; $46 \%$ Female) were included in the analysis. Baseline characteristics are presented in Table 1. All subjects demonstrated signs and symptoms of HF, elevated NTproBNP and objective evidence of LV systolic and/or diastolic function. Echocardiographic and invasive data of the cohort is presented in Table 2. LV EF was reduced $(<50 \%)$ in 55 (49\%) of the patients. Patients had elevated filling pressures represented by elevated mitral E/e', dilated LA volumes and elevated PA systolic pressure. Further, the cohort demonstrated elevated PA systolic, diastolic, mean pressures $\left(\mathrm{PAP}_{\mathrm{S}}, \mathrm{PAP}_{\mathrm{D}}\right.$, and $\mathrm{PAP} \mathrm{P}_{\mathrm{M}}$ respectively), mean pulmonary capillary wedge pressures $\left(P A W P_{M}\right)$ and increased pulmonary vascular resistance (PVR) on RHC. Sixty-five patients (58\%) demonstrated $\mathrm{PH}$, as defined by $\mathrm{PAP}_{\mathrm{M}} \geq 25 \mathrm{~mm} \mathrm{Hg}$ in keeping with current recommendations. Seventy-eight patients (70\%) demonstrated $\mathrm{PH}$ when the revised $\mathrm{PAP}_{\mathrm{M}}>20 \mathrm{~mm} \mathrm{Hg}$ cut-off was considered.

\subsection{Feasibility and accuracy of doppler PAP $_{M}$ algorithms}

Echocardiographic assessment of $\mathrm{PAP}_{\mathrm{M}}$ was most feasible employing the approach considering RVOT $_{A T}$ introduced by Dabestani et al. ${ }^{14}$ ( $86 \%$ of patients could have PAP $_{M}$ assessed using this method), followed by TR-derived assessments by Chemla et al. ${ }^{10}$ (84\%) and Aduen 
TABLE 1 Characteristics of patient population

\begin{tabular}{|c|c|}
\hline & $\begin{array}{l}\text { All patients } \\
(n=112)\end{array}$ \\
\hline \multicolumn{2}{|l|}{ Demographics } \\
\hline Age (years) & $60 \pm 16$ \\
\hline Female & $51(46)$ \\
\hline \multicolumn{2}{|l|}{ Medical history } \\
\hline Diabetes & $20(18)$ \\
\hline Hypertension & $68(61)$ \\
\hline Hypercholesteremia & $36(32)$ \\
\hline \multicolumn{2}{|l|}{ NYHA Class } \\
\hline \multirow[t]{2}{*}{ I/II/III/IV } & $11 / 18 / 77 / 6$ \\
\hline & $(10 / 16 / 69 / 5)$ \\
\hline \multicolumn{2}{|l|}{ Clinical assessment } \\
\hline Heart rate (bpm) & $69 \pm 14$ \\
\hline Body surface area $\left(\mathrm{m}^{2}\right)$ & $1.92 \pm .2$ \\
\hline Systolic blood pressure (mm Hg) & $117 \pm 24$ \\
\hline Diastolic blood pressure (mm Hg) & $66 \pm 13$ \\
\hline \multicolumn{2}{|l|}{ Laboratory } \\
\hline NTproBNP (ng/L) & $1450(395: 2730)$ \\
\hline Hemoglobin (g/L) & $131 \pm 98$ \\
\hline Serum creatinine ( $\mu \mathrm{mol} / \mathrm{L})$ & $98 \pm 37$ \\
\hline \multicolumn{2}{|l|}{ Diagnosis } \\
\hline Restrictive cardiomyopathy & $9(8)$ \\
\hline Dilated cardiomyopathy & $30(27)$ \\
\hline Hypertrophic cardiomyopathy & $4(4)$ \\
\hline Ischemic cardiomyopathy & $17(15)$ \\
\hline Multifactorial & $48(43)$ \\
\hline Myocarditis & $4(3)$ \\
\hline
\end{tabular}

Data presented as mean $\pm \mathrm{SD}$ / median (Q1;Q3) or number (\%).

Abbreviations: NYHA, New York heart association function class; NTproBNP $\mathrm{N}$-terminal pro b-type natriuretic peptide.

et al. $^{7}$ (81\%). PAP ${ }_{\mathrm{M}}$ estimated using the PR-derived approach (Abbas et al. ${ }^{9}$ ) was least feasible of the four methods (53\%). All echocardiographic $\mathrm{PAP}_{\mathrm{M}}$ algorithms demonstrated a moderately significant correlation with invasive variables $(r=.41-.65 ; p<0.001$ for all) (Figure S1). The method proposed by Aduen et al. ${ }^{7}$ demonstrated the strongest correlation with invasive $\operatorname{PAP}_{M}(r=.65 ; p<0.001)$, comparable with recommendation-based $\operatorname{TRV}_{\max }(r=.64 ; p<0.001)$. Agreement between each echocardiographic approach and RHC was studied using Bland-Altman analysis (Figure 2). Echocardiography demonstrated good accuracy to represent invasive pressures in the methods employing TR gradients (Aduen et al. ${ }^{7}$ and Chemla et al. ${ }^{10}$ ), as seen in relatively low bias between echocardiography and $\mathrm{RHC}$ (bias $=+2.4$ and $-2.4 \mathrm{~mm} \mathrm{Hg}$, respectively). Moderate precision was observed with limits of agreement (mean value $\pm 1.96 \times$ SD) in the range of $\pm 20 \mathrm{~mm}$ $\mathrm{Hg}$ for both methods. Relatively higher systematic error between diagnostic modalities was observed for approaches by Dabestani et al. ${ }^{14}$
TABLE 2 Invasive and echocardiographic data of patient population

\begin{tabular}{|c|c|}
\hline & $\begin{array}{l}\text { All } \\
(n=112)\end{array}$ \\
\hline \multicolumn{2}{|c|}{ Right heart catheterization } \\
\hline $\mathrm{PAWP}_{\mathrm{M}}(\mathrm{mm} \mathrm{Hg})$ & $17 \pm 8$ \\
\hline $\mathrm{PAP}_{\mathrm{S}}(\mathrm{mm} \mathrm{Hg})$ & $45 \pm 18$ \\
\hline $\mathrm{PAP}_{\mathrm{D}}(\mathrm{mm} \mathrm{Hg})$ & $18 \pm 9$ \\
\hline $\mathrm{PAP}_{\mathrm{M}}(\mathrm{mm} \mathrm{Hg})$ & $29 \pm 12$ \\
\hline $\mathrm{RAP}_{\mathrm{M}}(\mathrm{mm} \mathrm{Hg})$ & $8 \pm 6$ \\
\hline $\mathrm{RVP}_{\mathrm{S}}(\mathrm{mm} \mathrm{Hg})$ & $44 \pm 18$ \\
\hline $\mathrm{RVP}_{\mathrm{D}}(\mathrm{mm} \mathrm{Hg})$ & $10 \pm 6$ \\
\hline PVR (WU) & $2.7 \pm 1.9$ \\
\hline TPG (mm Hg) & $12 \pm 7$ \\
\hline $\mathrm{CO}(\mathrm{L} / \mathrm{min})$ & $4.7 \pm 1.6$ \\
\hline \multicolumn{2}{|l|}{ Echocardiography } \\
\hline \multicolumn{2}{|l|}{ Left heart } \\
\hline LVIDd (mm) & $55 \pm 13$ \\
\hline LVEDV (ml) & $157 \pm 90$ \\
\hline LV mass (gm) & $221 \pm 96$ \\
\hline LVEF (\%) & $46 \pm 20$ \\
\hline LV E/e' average & $14 \pm 6$ \\
\hline LAVI $\left(\mathrm{ml} / \mathrm{m}^{2}\right)$ & $47 \pm 17$ \\
\hline \multicolumn{2}{|l|}{ Right heart } \\
\hline RVIDd (mm) & $40 \pm 8$ \\
\hline TAPSE (mm) & $17 \pm 5$ \\
\hline $\mathrm{RVSP}(\mathrm{mm} \mathrm{Hg})$ & $48 \pm 17$ \\
\hline $\mathrm{RA}$ area $\left(\mathrm{cm}^{2}\right)$ & $20 \pm 7$ \\
\hline
\end{tabular}

Data presented as mean \pm SD or number (\%).

$p$-value represents difference between RA reservoir strain subgroups. Abbreviations: PAWP, pulmonary capillary wedge pressure, $\mathrm{PAP}_{\mathrm{M}}$, mean pulmonary artery pressure; $\mathrm{PAP}_{\mathrm{D}}$, diastolic pulmonary artery pressure; RAP, right atrial pressure; RVP, right ventricular pressure; PVR, pulmonary vascular resistance; TPG, transpulmonary gradient; $C O$, cardiac output; LVIDd, left ventricular internal diameter during end-diastole; LVEDV, left ventricular end-diastolic volume; $E F$, ejection fraction; RVID, right ventricular internal diameter end-diastole; TAPSE, tricuspid annular plane systolic excursion; TRV $_{\max }$, tricuspid regurgitation max velocity; RVSP, right ventricular systolic pressure; LAVI, left atrial volume index.

(that employed $\mathrm{RVOT}_{\mathrm{AT}}$ ) that overestimated invasive measurements (bias $=+4.2 \mathrm{~mm} \mathrm{Hg}$ ) and Abbas et al. ${ }^{9}$ (that employed PR peak velocity) that underestimated invasive measurements (bias $=-6.1 \mathrm{~mm} \mathrm{Hg}$ ). Relatively wider limits of agreement were seen in both algorithms.

\subsection{Diagnostic performance of echocardiographic algorithms to identify $\operatorname{PAP}_{M} \geq 25 \mathrm{~mm} \mathrm{Hg}$}

Recommendation-based $\mathrm{TRV}_{\max }$ demonstrated strong discriminatory ability to identify invasive $\operatorname{PAP}_{\mathrm{M}} \geq 25 \mathrm{~mm} \mathrm{Hg}$ (AUC $=.84, \mathrm{Cl}$ $.76-.91 ; p<0.001$ ) (Figure $3 \mathrm{~A}$ ). All echocardiographic approaches 

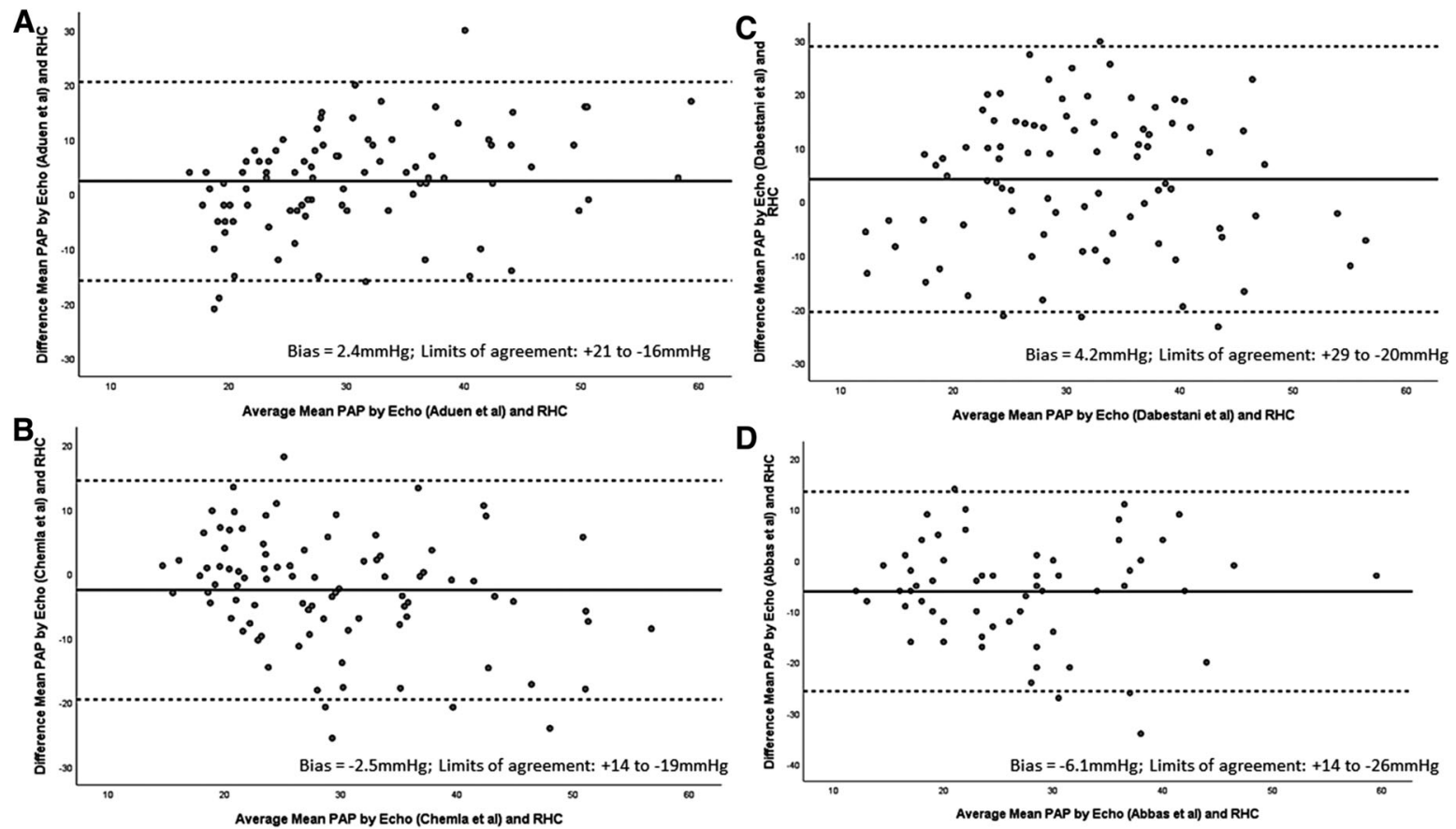

FIG URE 2 Bland-Altman plots illustrating agreement between echocardiographic algorithms and invasive PAPM measurements
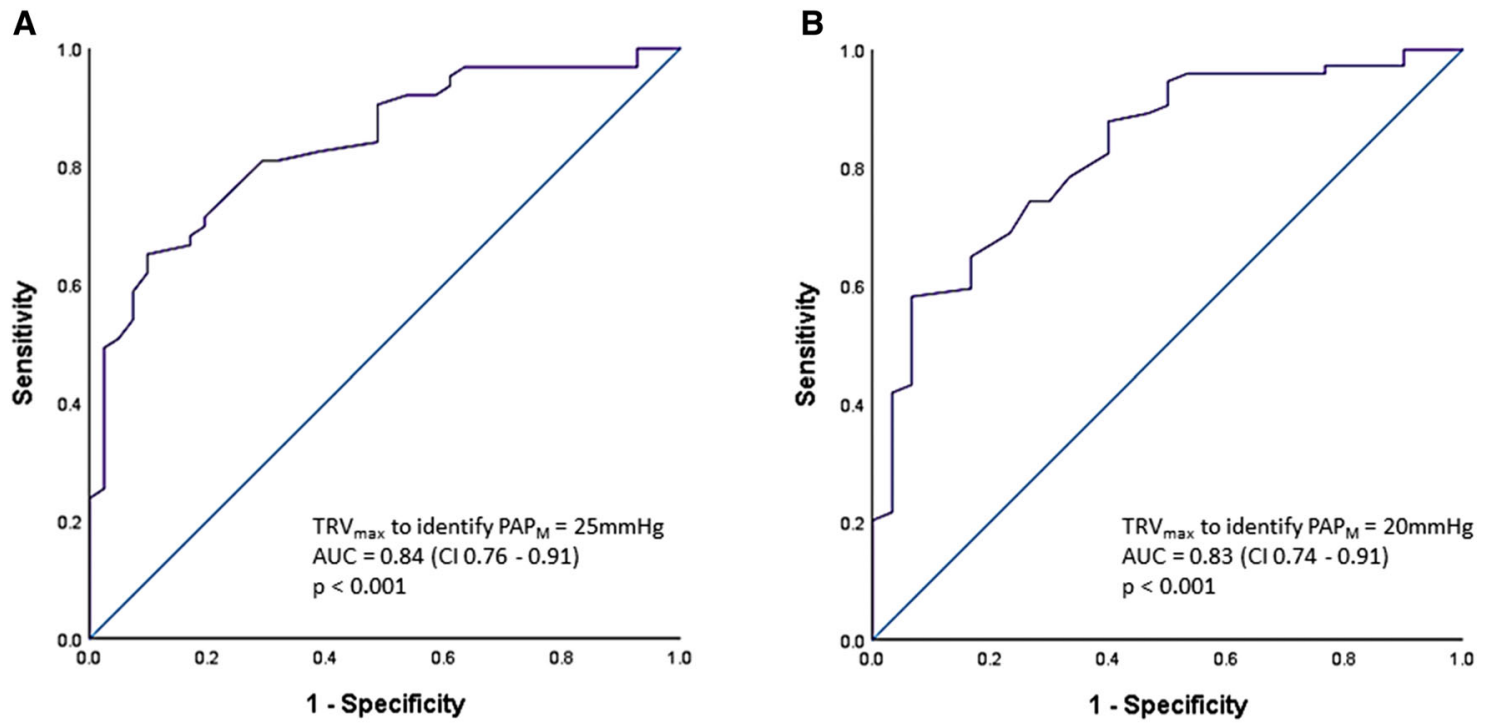

FIGURE 3 Receiver operator characteristics analysis illustrating diagnostic performance of $T_{R V} V_{\max }$ to identify (A) PAP $\geq 25 \mathrm{~mm} H g$ and (B) $\operatorname{PAP}_{M}>20 \mathrm{~mm} \mathrm{Hg}$

demonstrated moderate to strong discrimination (AUC range .70-.80; $p<0.001$ for all) with the Chemla et al. algorithm ${ }^{10}$ demonstrating strongest diagnostic performance (AUC $=.80, \mathrm{Cl} .71-.89 ; p<0.001$ ) (Figure 4). Sensitivity, Specificity, PPV, and NPV of TRV max $_{\text {ax }}$ and algorithms to identify invasive $\mathrm{PAP}_{\mathrm{M}} \geq 25 \mathrm{~mm} \mathrm{Hg}$ are presented in Table 3 . The recommended TRV $V_{\text {max }}$ cut-off of $2.8 \mathrm{~m} / \mathrm{sec}$ demonstrated $83 \%$ sensitivity and $61 \%$ specificity to identify $\mathrm{PAP}_{\mathrm{M}} \geq 25 \mathrm{~mm} \mathrm{Hg}$. At a cut-off of $25 \mathrm{~mm} \mathrm{Hg}$, PAP $\mathrm{M}$ derived by Aduen et al. ${ }^{7}$ and Dabestani et al. ${ }^{14}$ demonstrated low specificity (38\% and $35 \%$, respectively) and Abbas et al., ${ }^{9}$ low sensitivity (48\%). The only algorithm to show compara- ble, strong, balanced sensitivity and specificity was that proposed by Chemla et al. ${ }^{10}$ (78\% sensitivity and $67 \%$ specificity).

\subsection{Diagnostic performance of echocardiographic algorithms to identify PAP $_{M}>20 \mathrm{~mm} \mathrm{Hg}$}

When a revised, lowered $\mathrm{PH}$ cut-off was considered, TRV $\mathrm{max}_{\text {max }}$ continued to demonstrate strong diagnostic ability (AUC = .83, Cl .74-.91; $p<0.001$ ) (Figure 3B) when compared with other echocardiographic 

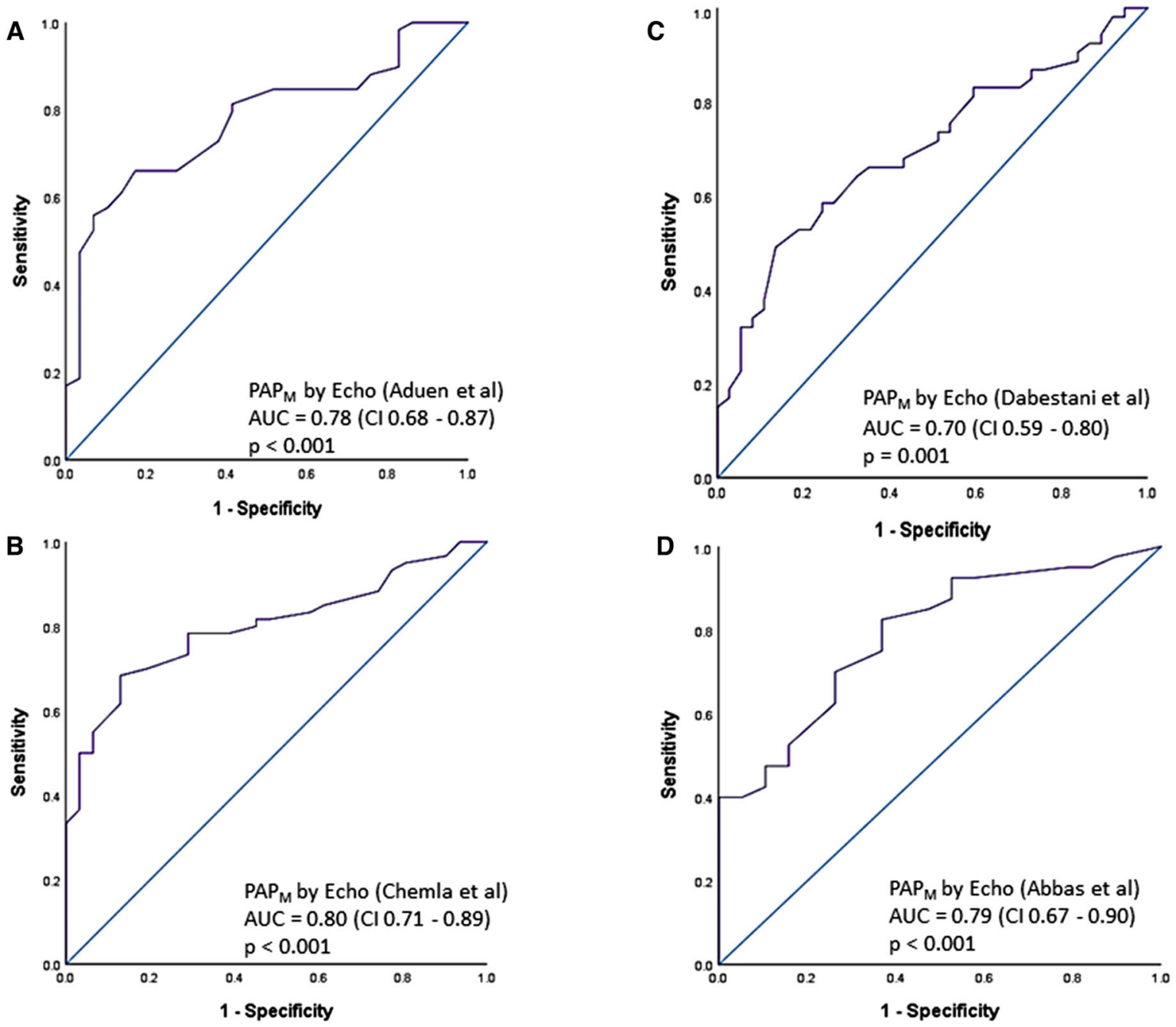

FIGURE 4 Receiver operator characteristics analysis illustrating diagnostic performance of echocardiographic algorithms to identify $P A P_{M} \geq$ $25 \mathrm{~mm} \mathrm{Hg}$

TAB LE 3 Sensitivity, specificity, positive predictive value, negative predictive value for recommendation-based cut-off in addition to echocardiographic algorithms to identify invasive PAP $_{M} \geq 25 \mathrm{~mm} \mathrm{Hg}$

\begin{tabular}{|c|c|c|c|c|c|}
\hline Method & Cut off & $\begin{array}{l}\text { Sensitivity } \\
\text { (\%) }\end{array}$ & Specificity(\%) & $\begin{array}{l}\text { Positive } \\
\text { predictive } \\
\text { value (\%) }\end{array}$ & $\begin{array}{l}\text { Negative } \\
\text { predictive value } \\
\text { (\%) }\end{array}$ \\
\hline Aduen et al. & $25 \mathrm{~mm} \mathrm{Hg}$ & 85 & 38 & 74 & 55 \\
\hline Chemla et al. & $25 \mathrm{~mm} \mathrm{Hg}$ & 78 & 67 & 80 & 63 \\
\hline Dabestani et al. & $25 \mathrm{~mm} \mathrm{Hg}$ & 83 & 35 & 63 & 61 \\
\hline
\end{tabular}

approaches (AUC range .67-.71; $p<0.05$ ) (Figure S2). At a cut-off of $2.8 \mathrm{~m} / \mathrm{sec}, \mathrm{TRV}_{\max }$ demonstrated $78 \%$ sensitivity and $68 \%$ specificity to identify invasive $\mathrm{PAP}_{\mathrm{M}}>20 \mathrm{~mm} \mathrm{Hg}$. Specificity was generally low for all echocardiographic methods (17\%-35\%) with the exception of the algorithm proposed by Abbas et al. ${ }^{9}$ (64\% sensitivity, $67 \%$ specificity) (Table S1)

\subsection{Accuracy of echocardiographic right atrial pressure estimates}

Echocardiographic RAP employing IVC size and collapse were incorporated to calculate $\mathrm{PAP}_{\mathrm{M}}$ in all echocardiographic algorithms with the exception of the approach postulated by Dabestani et al. ${ }^{12}$ In 107 
subjects (96\%) with interpretable images, RAP estimated by IVC was elevated ( 8 or $15 \mathrm{~mm} \mathrm{Hg}$ ) in $78 \%$ subjects ( $n=83$, RAP $=8 \mathrm{~mm} \mathrm{Hg}$ in 43 and $15 \mathrm{~mm} \mathrm{Hg}$ in 40 subjects). However, false positives were frequent, as seen in 12 of 40 patients (30\%) with significantly elevated RAP estimated by echocardiography $(15 \mathrm{~mm} \mathrm{Hg}$ ) that had normal invasive RAP ( $\leq 7 \mathrm{~mm} \mathrm{Hg}$ ).

\section{4 | DISCUSSION}

To the best of our knowledge, this is the first study to evaluate the accuracy of multiple echocardiographic algorithms to estimate $\mathrm{PAP}_{\mathrm{M}}$ and study diagnostic performance to identify $\mathrm{PH}$ in the specific setting of HF. All four echocardiographic algorithms demonstrated reasonable association and good agreement with RHC, with generally lower bias seen in algorithms considering tricuspid mean or peak gradient. However, none of the echocardiographic algorithms outperformed $T_{R} V_{\text {max }}$ to identify $\mathrm{PH}$ in the $\mathrm{ROC}$ analysis.

The accuracy of echocardiographic algorithms to estimate pulmonary artery pressures has been a matter of debate. Earlier studies suggest that echocardiography frequently over- or underestimates invasive pulmonary pressures and should not be relied upon. ${ }^{16,17}$ More recent studies, however, have emphasized results of Bland-Altman analyses that display low bias between echocardiographic and invasive measurements, suggesting that Doppler estimates are highly accurate for population studies. ${ }^{18,19}$ Our data suggests that accuracy of echocardiographic estimates may also vary based on approach utilized. Minimal bias was observed in methods that incorporated TRV $V_{\max }$, corroborating an earlier study employing high-fidelity catheters that suggests that the most accurate estimate of PAPM $_{M}$ is obtained by assessment of systolic PA pressures alone ${ }^{20}$ Higher systemic bias with invasive measurements and lower precision reflected in wider limits of agreement employing both PI (Abbas et al. ${ }^{9}$ ) and RVOT AT $_{\text {(Dabestani }}$ et al. ${ }^{14}$ ) seen in this study may, at least in part, be attributable to smaller patient cohorts ( $n=23$ and 39, respectively) and less severe clinical presentations in the original studies. As seen in the Bland-Altman plots, a greater dispersion of points is observed at higher mean values of $\mathrm{PAP}_{\mathrm{M}}$, suggesting that these methods may be less reliable in the setting of severe $\mathrm{PH}$. The cohort examined by Abbas et al. demonstrated a $\mathrm{PAP}_{\mathrm{M}}=25$ (range 10-57) $\mathrm{mm} \mathrm{Hg}$ and $\mathrm{PAWP}_{\mathrm{M}}=15$ (range 2-38) $\mathrm{mm} \mathrm{Hg}$, suggesting a milder hemodynamic presentation compared with cohort in our study. ${ }^{9}$ Dabestani et al. do not present corresponding values in their cohort, but suggest a $\mathrm{PAP}_{\mathrm{M}}$ range, that is, relatively lower than that in our study with lower PH cut-off $(20 \mathrm{~mm} \mathrm{Hg}){ }^{14}$ Additionally, the empirical algorithms presented using this method may demonstrate limited utility in the setting of severely elevated $\mathrm{PAP}_{\mathrm{M}}$, as alluded to in certain comparative studies evaluating multiple echocardiographic approaches. ${ }^{21}$ Despite displaying relatively lower precision and agreement with invasive measurements, both the abovementioned methods demonstrated good diagnostic ability to identify $\mathrm{PH}$ in our cohort. Uninterpretable TR signals are frequent in $\mathrm{HF}^{22}$ have been reported in as many as $39 \%$ of subjects and may present a limitation to echocardiographic evaluation of $\mathrm{PH}^{23}$ In our study, $\mathrm{TRV}_{\max }$ could not be adequately assessed in $14 \%$ and velocity-time integral in $19 \%$ of patients, emphasizing the diagnostic role for methods that do not necessitate TR jet interrogation.

Early identification of $\mathrm{PH}$ in $\mathrm{HF}$ has direct consequences on treatment and prognosis. Despite reasonable to strong diagnostic ability to identify $\mathrm{PH}$, none of the echocardiographic algorithms outperformed $T R V_{\text {max }}$ on ROC analysis, irrespective of hemodynamic definition of $\mathrm{PH}$. This finding is contrary to a recent comparative report where the chosen echocardiographic algorithms showcased generally superior performance as compared with $\mathrm{TRV}_{\max } \cdot{ }^{21}$ The authors suggest in the abovementioned study that algorithms that consider estimates of right atrial pressure in addition to $\mathrm{TRV}_{\text {max }}$ demonstrate generally stronger correlation with invasive measurements and superior diagnostic performance when compared with $\mathrm{TRV}_{\max }$ alone. This was substantiated by data from their study where right atrial pressure $>15 \mathrm{~mm} \mathrm{Hg}$ estimated by echocardiography demonstrated highest odds ratio for invasively confirmed $\mathrm{PH}$. In the setting of HF, echocardiographic estimates of right atrial pressure are frequently falsely elevated and sole reliance on the IVC to estimate RAP may be misleading. ${ }^{24}$ In our study, close to one in three patients with elevated RAP estimated by echocardiography demonstrated normal corresponding invasive pressures suggesting that these estimates are frequently inaccurate and may not necessarily contribute to stronger performance of derived $\mathrm{PAP}_{\mathrm{M}}$ variables as earlier suggested..$^{9,21}$ Echocardiographic estimates of RAP $_{M}$ have been incorporated into empirical derivations of $\mathrm{PAP}_{\mathrm{M}}$ in all but one selected $\mathrm{PAP}_{\mathrm{M}}$ algorithms in this study. This may play a role in the observed lower performance when compared with $\mathrm{TRV}_{\max }$ alone, but needs to be further examined.

The use of fluid-filled catheters instead of high-fidelity manometertipped catheters for pressure measurement might introduce additional error and may be considered a limitation in this study. Retrospective analysis of echocardiographic data did not permit a closer inspection factors leading to lower feasibility of certain algorithms included in this comparative analysis. Finally, we did not employ agitated saline bubble contrast to strengthen TR jet signal as this is not part of routine protocol in our laboratory.

\section{5 | CONCLUSIONS}

In the setting of $\mathrm{HF}$, echocardiographic estimates of $\mathrm{PAP}_{\mathrm{M}}$ are highly feasible, demonstrate reasonable association and good agreement with invasive measurements. Despite displaying strong ability to identify $\mathrm{PH}$, none of the methods outperformed recommendationproposed TRV $V_{\max }$ cut-off $>2.8 \mathrm{~m} / \mathrm{sec}$.

\section{ACKNOWLEDGMENT}

Ashwin Venkateshvaran is supported by grants from the Swedish Association for pulmonary hypertension; Lars $\mathrm{H}$. Lund is supported by grants from the Swedish Research Council [grants 2013-23897104604-23 and 523-2014-2336], Swedish Heart Lung Foundation [grants 20100419 and 20120321], Stockholm County Council [grants 20110120 and 20140220] and Swedish Society of Medicine [grants 174111 and 504881]. 


\section{CONFLICT OF INTEREST}

All authors declare no conflicts of interests related to this study.

\section{ORCID}

Ashwin Venkateshvaran PhD (DD https://orcid.org/0000-0002-0676-

0604

\section{REFERENCES}

1. Guazzi M, Borlaug BA. Pulmonary hypertension due to left heart disease. Circulation. 2012;126(8):975-990.

2. Rosenkranz S, Gibbs JSR, Wachter R, et al. Left ventricular heart failure and pulmonary hypertension. Eur Heart J. 2016;37(12):942-954.

3. Rao SD, Adusumalli S, Mazurek JA. Pulmonary hypertension in heart failure patients. Cardiac Fail Rev. 2020;6.

4. Galiè N, Humbert M, Vachiery J-L, et al. 2015 ESC/ERS guidelines for the diagnosis and treatment of pulmonary hypertension: the Joint Task Force for the Diagnosis and Treatment of Pulmonary Hypertension of the European Society of Cardiology (ESC) and the European Respiratory Society (ERS): endorsed by: association for European Paediatric and Congenital Cardiology (AEPC), International Society for Heart and Lung Transplantation (ISHLT). Eur Heart J. 2015;37(1):67-119.

5. Simonneau G, Montani D, Celermajer DS, et al. Haemodynamic definitions and updated clinical classification of pulmonary hypertension. Eur Respir J. 2019;53(1).

6. Maron BA, Brittain EL, Choudhary G, Gladwin MT. Redefining pulmonary hypertension. Lancet Respir Med. 2018;6(3):168-170.

7. Aduen JF, Castello R, Lozano MM, et al. An alternative echocardiographic method to estimate mean pulmonary artery pressure: diagnostic and clinical implications. J Am Soc Echocardiogr. 2009;22(7):814819.

8. Kitabatake A, Inoue M, Asao M, et al. Noninvasive evaluation of pulmonary hypertension by a pulsed Doppler technique. Circulation. 1983;68(2):302-309.

9. Abbas AE, Fortuin FD, Schiller NB, et al. Echocardiographic determination of mean pulmonary artery pressure. Am J Cardiol. 2003;92(11):1373-1376.

10. Chemla D, Castelain V, Humbert M, et al. New formula for predicting mean pulmonary artery pressure using systolic pulmonary artery pressure. Chest. 2004;126(4):1313-1317.

11. Er F, Ederer S, Nia AM, et al. Accuracy of Doppler-echocardiographic mean pulmonary artery pressure for diagnosis of pulmonary hypertension. PLoS One. 2010;5(12):e15670.

12. Dabestani A, Mahan G, Gardin JM, et al. Evaluation of pulmonary artery pressure and resistance by pulsed Doppler echocardiography. Am J Cardiol. 1987;59(6):662-668.

13. Steckelberg RC, Tseng AS, Nishimura R, Ommen S, Sorajja P. Derivation of mean pulmonary artery pressure from noninvasive parameters. J Am Soc Echocardiogr. 2013;26(5):464-468.

14. Dabestani A, Mahan G, Gardin JM, et al. Evaluation of pulmonary artery pressure and resistance by pulsed Doppler echocardiography. Am J Cardiol. 1987;59(6):662-668.
15. Lang RM, Badano LP, Mor-Avi V, et al. Recommendations for cardiac chamber quantification by echocardiography in adults: an update from the American Society of Echocardiography and the European Association of Cardiovascular Imaging. Eur Heart J Cardiovasc Imag. 2015;16(3):233-270

16. Fisher MR, Forfia PR, Chamera E, et al. Am J Respir Crit Care Med. 2009;179(7):615-621.

17. Rich JD, Shah SJ, Swamy RS, Kamp A, Rich S. Inaccuracy of Doppler echocardiographic estimates of pulmonary artery pressures in patients with pulmonary hypertension: implications for clinical practice. Chest. 2011;139(5):988-993.

18. D'alto $M$, Romeo $E$, Argiento $P$, et al. Accuracy and precision of echocardiography versus right heart catheterization for the assessment of pulmonary hypertension. Int J Cardiol. 2013;168(4):40584062.

19. Venkateshvaran A, Seidova N, Tureli HO, et al. Accuracy of echocardiographic estimates of pulmonary artery pressures in pulmonary hypertension: insights from the KARUM hemodynamic database. Int J Cardiovasc Imag. 2021:1-9.

20. Chemla D, Castelain V, Provencher S, Humbert M, Simonneau G, Hervé $P$. Evaluation of various empirical formulas for estimating mean pulmonary artery pressure by using systolic pulmonary artery pressure in adults. Chest. 2009;135(3):760-768.

21. Hellenkamp K, Unsöld B, Mushemi-Blake S, et al. Echocardiographic estimation of mean pulmonary artery pressure: a comparison of different approaches to assign the likelihood of pulmonary hypertension. J Am Soc Echocardiogr. 2018;31(1):89-98.

22. Faxen UL, Venkateshvaran A, Shah SJ, et al. Generalizability of HFAPEFF and H2FPEF diagnostic algorithms and associations with heart failure indices and proteomic biomarkers: insights from pROMISHFpEF. J Cardiac Fail. 2021.

23. Amsallem M, Sternbach JM, Adigopula S, et al. Addressing the controversy of estimating pulmonary arterial pressure by echocardiography. J Am Soc Echocardiogr. 2016;29(2):93-102.

24. Miah N, Faxén UL, Lund LH, et al. Diagnostic utility of right atrial reservoir strain to identify elevated right atrial pressure in heart failure. Int J Cardiol. 2020.

\section{SUPPORTING INFORMATION}

Additional supporting information may be found online in the Supporting Information section at the end of the article.

How to cite this article: Björkman A, Lund LH, Faxén UL, Lindquist $P$, Venkateshvaran A. Accuracy and diagnostic performance of doppler echocardiography to estimate mean pulmonary artery pressure in heart failure. Echocardiography. 2021;38:1624-1631. https://doi.org/10.1111/echo.15188 